\title{
Artelogie
}

Recherche sur les arts, le patrimoine et la littérature de I'Amérique latine

$11 \mid 2017$

Délocalités, translocalités et activisme dans l'art électronique et biomédiale latino-américain

\section{À propos du livre de Sonia Guggisberg sur le documentaire vidéo}

Jacques Leenhardt

\section{OpenEdition}

Journals

Édition électronique

URL : http://journals.openedition.org/artelogie/1611

DOI : 10.4000/artelogie.1611

ISSN : 2115-6395

Éditeur

Association ESCAL

Référence électronique

Jacques Leenhardt, « À propos du livre de Sonia Guggisberg sur le documentaire vidéo », Artelogie [En ligne], 11 | 2017, mis en ligne le 28 décembre 2017, consulté le 24 septembre 2020. URL : http:// journals.openedition.org/artelogie/1611; DOI : https://doi.org/10.4000/artelogie.1611

Ce document a été généré automatiquement le 24 septembre 2020

Association ESCAL 


\title{
À propos du livre de Sonia Guggisberg sur le documentaire vidéo
}

\author{
Jacques Leenhardt
}

1 Le sous-titre de l'ouvrage que propose Sonia Guggisberg, Réseaux d'images, mémoires et témoignages: pour une documentation performative des savoirs ${ }^{1}$ annonce clairement le projet qui s'y trouve exposé. Mais que faut-il entendre par «documentation performative des savoirs »? Sonia Guggisberg est une vidéaste et artiste multimédia brésilienne. Elle part de son travail de terrain et s'interroge sur les conditions (esthétiques d'abord, mais aussi techniques et politiques) qui donneront à ce travail documentaire son efficacité, sa puissance performative. La capacité à toucher les spectateurs ne dépend donc pas des seuls faits rapportés. Elle résulte au contraire d'une construction qui engage l'artiste mais surtout de la manière dont il saura articuler les différents savoirs qui sont plus ou moins spontanément mis en œuvre par son travail.

2 Une «documentation performative des savoirs» est donc le résultat d'un travail critique et réfléchi sur la prise de vue, sur l'enregistrement et la restitution de la bande son, sur la sélection des points de vue et sur le rôle que jouent les différents savoirs historiques, sociologiques ou anthropologique dans l'élaboration de chacun de ces choix. Sonia Guggisberg s'en tient au plan du travail de l'artiste, de la poḯtique de sa mise en œuvre, sans aborder un autre continent de questions qui déterminent évidemment aussi la capacité performative de la documentation : l'usage des canaux institutionnels de diffusion. On la suivra donc sur le terrain qu'elle s'est choisi ainsi que sur le retour sur expérience qu'elle propose plutôt qu'un exposé théorique. Les différentes vidéos qu'elle a réalisées seront la pierre de touche de cet examen.

Partant de l'idée que tout travail artistique qui s'attache à un événement ou une situation doit être considéré comme une traduction, l'artiste s'interroge d'abord sur cette étrange situation où l'artiste, confronté à des documents, des souvenirs, des archives, tous objets à l'élaboration desquels sont attachés des savoirs et des techniques, "traduit» ces objets et ces savoirs en une proposition artistique. Cette 
dernière s'annonce par ailleurs fortement politique et engagée dans la mesure où elle est liée à la volonté de rendre le spectateur conscient des enjeux abordés, et cela dans la perspective d'agir finalement sur la réalité sociale et politique. Il faut donc ranger Sonia Guggisberg dans l'ample famille des artistes critiques, qui conçoivent leur travail comme une manière spécifique de participer à la transformation du monde.

Une archéologie du contemporain

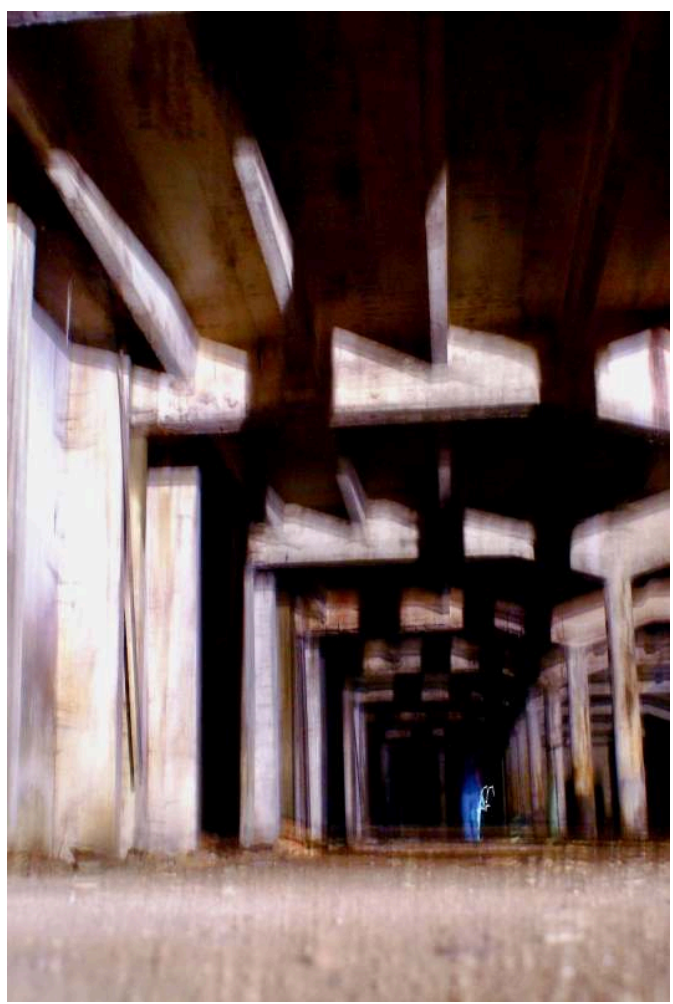

4 L'ouvrage commence par la présentation d'une vidéo documentaire réalisée sur un projet urbanistique des années 70 qui concernait la transformation radicale de l'Avenida Paulista, artère centrale de la ville de São Paulo (Brésil). Le titre de cette vidéo, Subsolo (2013), renvoie aux travaux souterrains qui avaient été nécessaires pour engager le projet de la Nouvelle Avenida Paulista et qui constituent aujourd'hui les traces visibles du projet urbain. Mais avant d'en apprendre davantage sur le sujet, il faut se mettre d'accord sur ce que signifie ici la notion de « documentaire ».

5 Sonia Guggisberg relève d'emblée que le genre documentaire a changé de statut à partir du moment où la culture contemporaine a pris acte de la nécessité de donner voix au chapitre à la diversité des points de vues et des modes de connaissance à partir desquels nous approchons les réalités dans lesquelles nous vivons. Cette acceptation de la multiplicité des regards a changé la notion même de documentaire. S'il fut un temps où l'on a cru que les documents qui constituent les documentaires étaient susceptibles à eux seuls d'atteindre à la vérité de ce qui s'est passé, cette époque est révolue et le chercheur tente aujourd'hui plutôt d'articuler différents savoirs et différentes approches. Il se propose dès lors non pas d'offrir une vérité, qu'il aura prétendu saisir en tant que témoin oculaire, mais un corpus d'images et de commentaires à entrées multiples à partir duquel chacun sera appelé à construire sa propre conviction et éventuellement, dans un deuxième temps, d'agir. 
Dans cet ouvrage, il ne s'agit pas pour Sonia Guggisberg d'entrer plus avant dans la problématique du caractère relatif de la vérité, qui agite depuis des décennies les débats entre philosophes et historiens. Elle cherche plutôt à explorer les exigences qui s'imposent à la volonté de montrer, de dire et de critiquer, voire de dénoncer. Elle part donc du principe que sa démarche doit prendre en compte la diversité sociale, politique et épistémique des moyens de connaître (savoirs, sentiments, révoltes) et que, de cette reconnaissance, dépend la poétique qui sera mise en œuvre et l'efficacité atteinte.

7 Alors venons-en à ce qui constitue le premier objet et le cœur de son livre: la Nouvelle Avenue Pauliste ou plutôt ce qu'il en reste, à savoir ses soubassements, qui expliquent le titre de l'œuvre : Subsolo.

Titre de l'œuvre

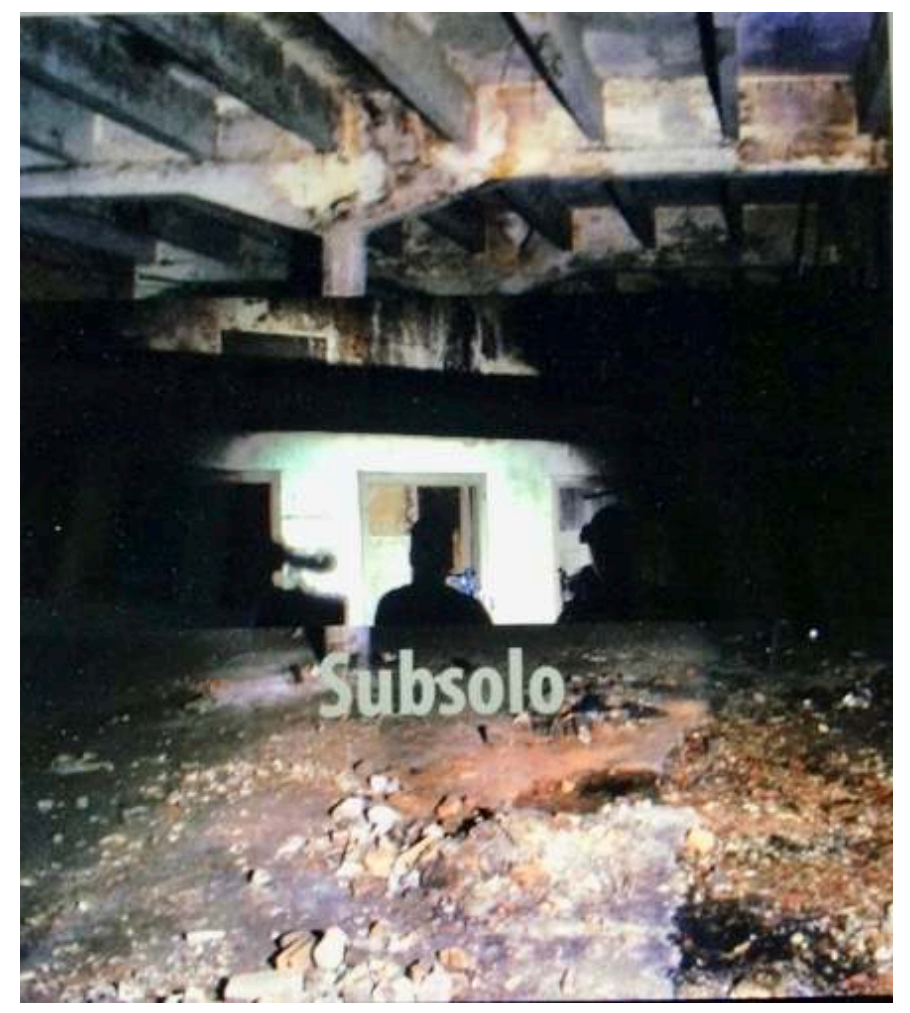

Dans les années soixante-dix, en pleine dictature militaire au Brésil, un vaste projet urbanistique est mis en œuvre pour la ville de São Paulo. Il s'agit de doubler souterrainement l'Avenida Paulista) qui en constitue l'axe majeur, laquelle se trouve saturée par la circulation automobile et les transports en commun de surface. Aux yeux de la municipalité, cette situation justifie l'idée de doubler cette voie qui relie en outre deux autres axes majeurs de la cité. Cette préoccupation est conforme à la logique urbanistique de l'époque, qui privilégie la fluidité des transports urbains et ne remet pas en cause l'usage individuel de l'automobile. Les promoteurs du projet justifient par ailleurs la création de cette deuxième voie de circulation enterrée en arguant que celleci permettra d'y concentrer le trafic de transit, dégageant ainsi en surface pour les habitants des espaces piétonniers, des aires de repos et des voies de circulation locale qui seront aménagés au niveau actuel de l'avenue. Un tunnel inférieur était également prévu pour un métro, ce qui allégerait d'autant la circulation à l'air libre. 
Or ce «beau » projet qui voulait redonner une place aux piétons en même temps qu'il imaginait une requalification du tissu urbain, n'a pas été mené à son terme. Interrompu après quelques années de travaux préparatoires destinés à reconfigurer les réseaux et les infrastructures du quartier, le projet de la Nova Avenida Paulista a été abandonné et son concepteur, l'ex-préfet Figueiredo Ferraz, destitué par le régime militaire.

10 Aujourd'hui, la ville a presque complètement perdu le souvenir du projet urbain de Ferraz et plus encore des gigantesques aménagements qui avaient bel et bien été réalisés. Sonia Guggisberg est donc partie à la recherche de cet objet urbain perdu, de ce Subsolo, occulté au regard et exclu des mémoires. Elle qui est née dans cette ville peu avant les évènements qu'elle tente de reconstruire, c'est aussi sa propre histoire et les circonstances de sa propre existence qui, sans doute, ont motivé cette quête d'une mémoire perdue.

Présence de l'artiste

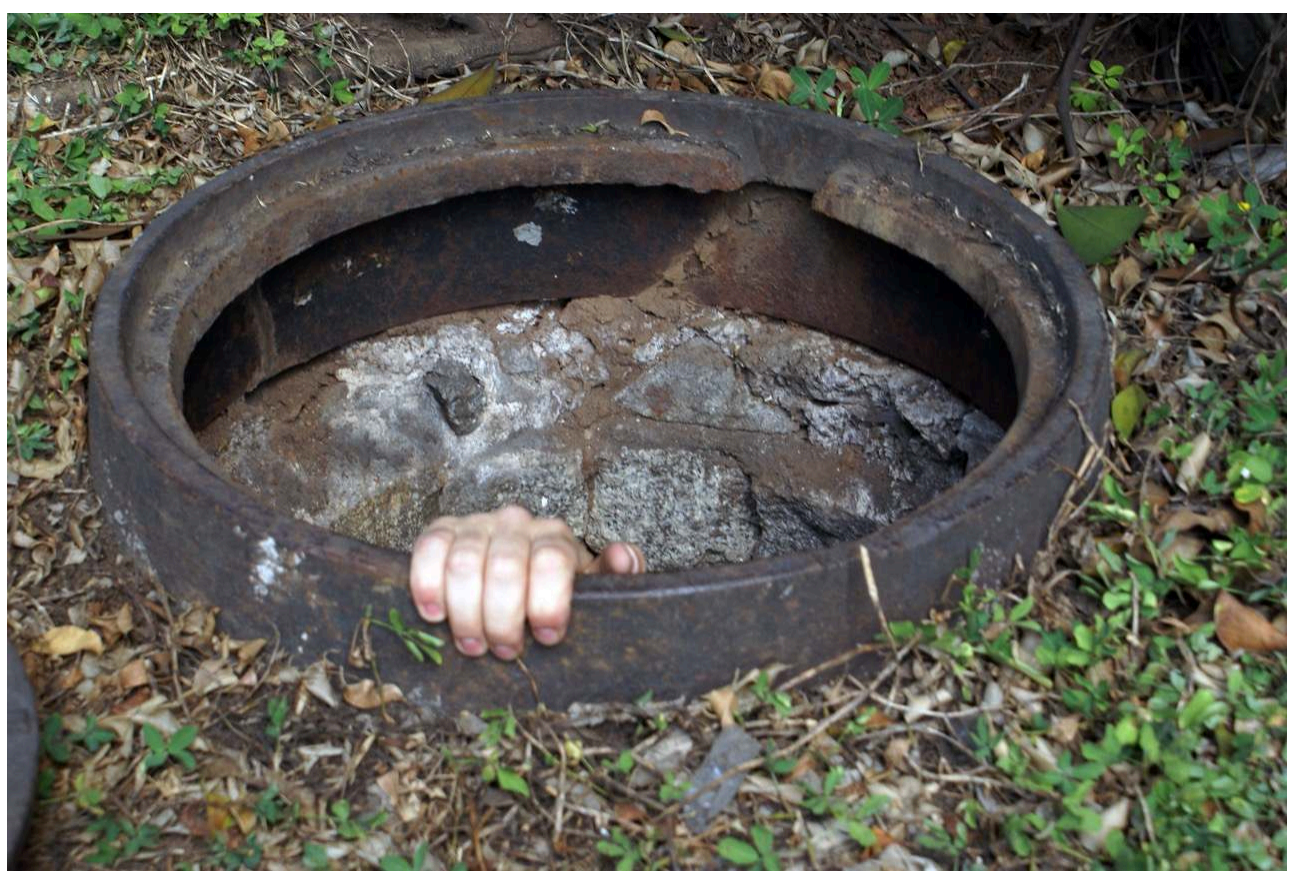

11 La vidéo que tourne Sonia Guggisberg entreprend donc de restituer aux nouvelles générations et à la ville elle-même qui souffre toujours d'une sorte de thrombose circulatoire, la mémoire effacée de cette transformation, à la fois espoir et traumatisme. Dans cette opération de reconstruction mémorielle, elle se trouve confrontée à un moment historique majeur du projet urbain de São Paulo que son abandon a transformé en un site archéologique contemporain.

On voit bien de quelle manière un tel objet, dans sa complexité, se prête à une réflexion sur l'articulation des mémoires et la multiplicité des savoirs. Comment en effet rendre compte de tant de voix, d'images, de documents et de souvenirs alors que l'histoire a plongé cette époque de la dictature dans le Léthé de la mémoire citoyenne.

13 Sonia Guggisberg détaille parfaitement les difficultés auxquelles elle se trouve confrontée: pour traiter de ce fantôme qui hante les profondeurs de la ville contemporaine, il faudra retrouver et articuler des discours et des souvenirs, des débats et des non-dits. Certes, de nombreux documents d'archives sont disponibles et la presse de l'époque a amplement rendu compte des intentions proclamées par les édiles, 
mais tout cela est désormais difficile à rassembler et délicat à hiérarchiser compte tenu du temps écoulé et de l'oubli dans lequel ces traces sommeillent.

Une vision d'architecte

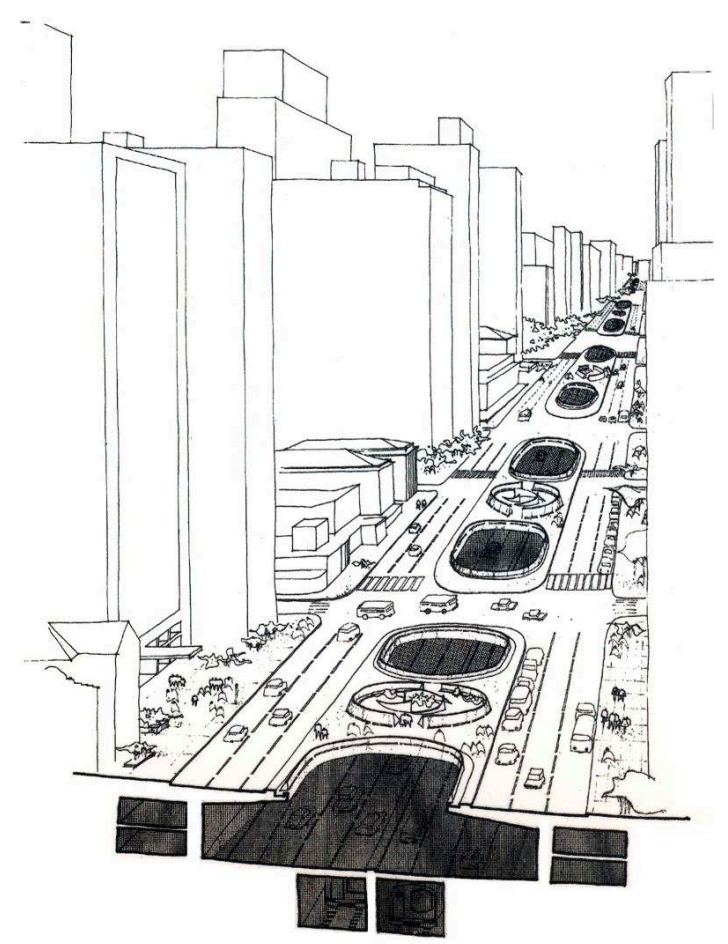

Il faudra donc que l'artiste entreprenne de confronter systématiquement ces traces matérielles aux archives des urbanistes et aux plans des architectes, à la presse de l'époque et à ce qui demeure dans la mémoire des acteurs qui sont encore nombreux à être vivants au moment où elle entreprend son enquête. Sur des photos d'époque, on verra ainsi des embouteillages et des immeubles flambant neufs, dont on sait par ailleurs qu'ils promettent de multiplier encore la densité du trafic.

De quelles vérités et de quels mensonges ces documents sont-ils les témoins et les agents? Comment les accorder aux ruines lacérées de rouille que la camera vidéo enregistre dans sa plongée souterraine? Et les acteurs de l'époque, comment expliquent-ils leur enthousiasme ou leurs doutes, et surtout comment parviennent-ils aujourd'hui à faire comprendre le contexte qui permit l'éclosion du projet et causa l'échec final de l'entreprise? Quelles furent les figures techniques, médiatiques ou politiques qui eurent un rôle déterminant, à moins que ceux dont ils ont gardé la mémoire n'aient été que des pions ou des faux-nez dans un jeu qui se déroulait sur d'autres plans, dans les couches plus secrètes de la gestion du projet, voire dans l'inconscient urbain et politique? 


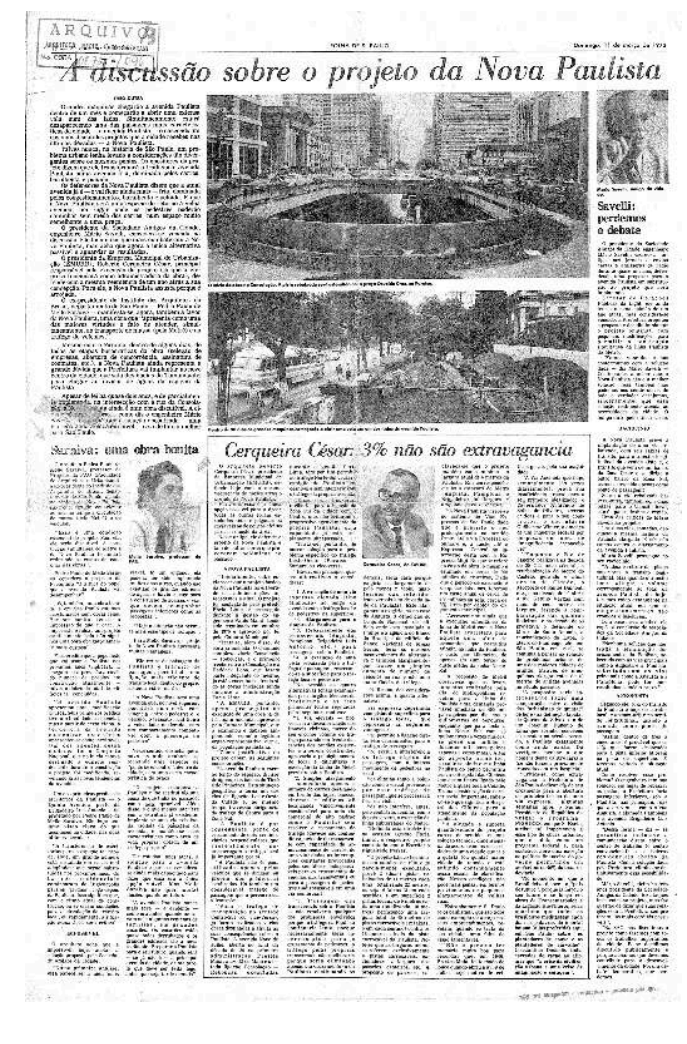

On voit que le travail de l'artiste documentariste joue avec un feuilleté de couches de signification d'une extrême variété, et Sonia Guggisberg se donne pour tâche de réfléchir aux moyens à sa disposition pour organiser cette complexité et la penser comme un travail critique, conscient de lui-même. Au-delà des documents d'époque et des traces matérielles qu'elle transforme en images, il y a encore la question de l' " archive ", non pas simplement les produits de l'archivage des faits et des données mais bien, comme disait Foucault, l' «archive» comme un système d'énonciabilité discursive. L' " archive » comme ce qui peut être énoncé ? Dans cette grande affaire de la ville de São Paulo, on comprend que beaucoup de choses ne sont simplement pas énonçables, qu'elles ne le seront peut-être jamais. Mais c'est justement là qu'on attend le travail de l'artiste et sa volonté, à travers la poétique documentaire, de faire sentir ce qui n'est pas énonçable.

Les témoins et leurs témoignages également ont leur fragilité : la mémoire, bien sûre, toujours prompte à sombrer, mais aussi ce biais que constitue la volonté des acteurs de reconstruire une histoire dans laquelle ils puissent s'attribuer un rôle avantageux. Alors il faut confronter les discours, comparer les sources, confier au montage, comme Raymond Depardon l'a si bien illustré, la tâche de faire entendre, même les silences, la responsabilité de mettre en avant ou de laisser en retrait. Un documentaire vidéo est une construction à partir de fragments arrachés à des chaînes signifiantes relativement autonomes que l'artiste doit briser pour faire advenir cet acte performatif, cette efficacité cognitive qu'il a en vue : pour produire, comme dit Sonia Guggisberg, une « documentation performative des savoirs». 
Plonger dans la recherche

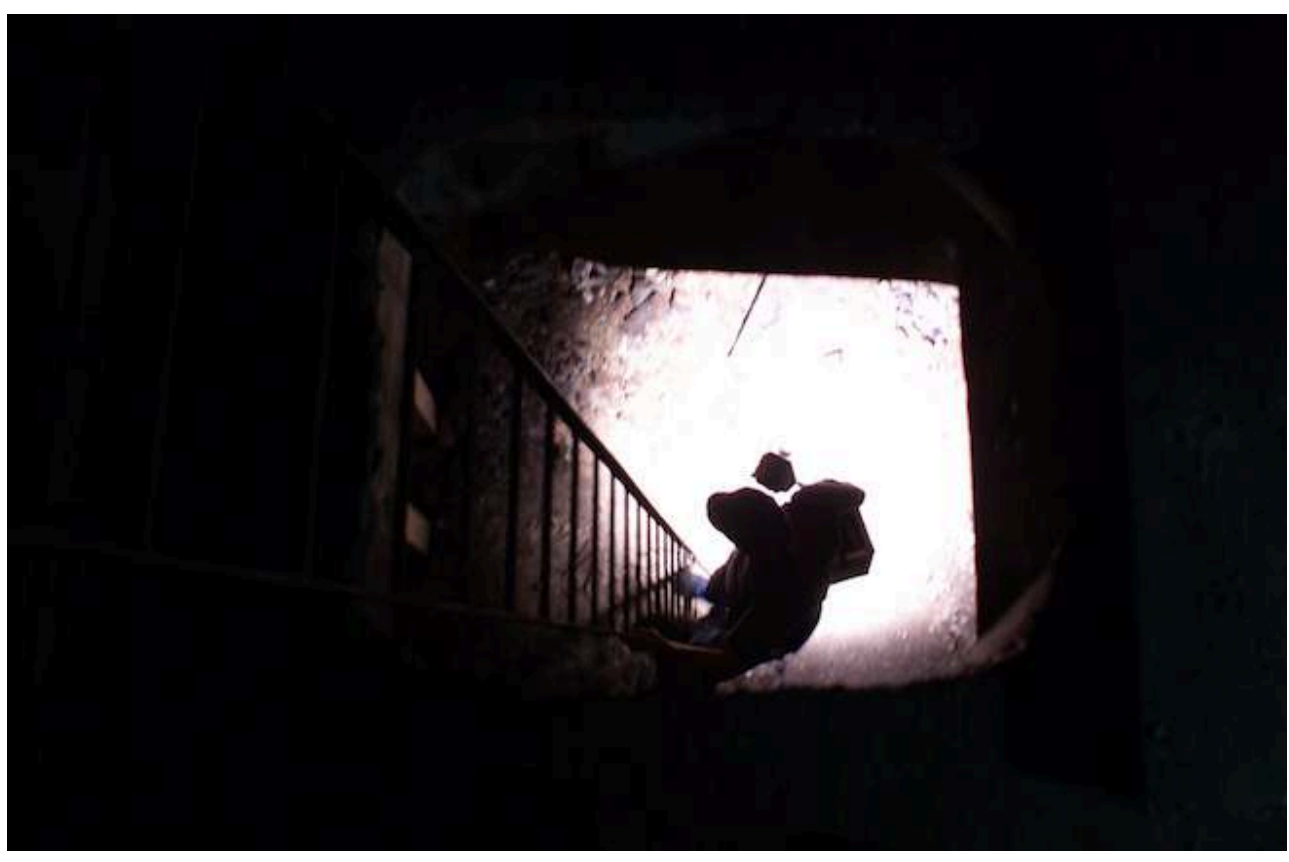

Faire entendre ce dont la voix a été étouffée, par le temps, par les intentions mauvaises ou simplement contradictoires, par la vie qui poursuit son cours, voilà l'exigence première. Rendre visible ce qui est caché, ce que l'inconséquence des édiles a enfoui sous des milliers de tonnes de macadam frais, porter la lumière dans les coins sombres du sous-sol et de l'urbanisme, voilà le projet exaltant de l'artiste documentariste qu'est Sonia Guggisberg. Mais il lui faudra encore se méfier de ce qui fait « image » et dévie le regard, comme aussi veiller à ce que la bandes-son permette aussi l'écoute de ce qui est presque inaudible, par delà les discours trop bien construits et démonstratifs.

Sonia Guggisberg travaille soigneusement ses enregistrements, elle sait leur imprimer la pertinence du contretemps, cet écart qui arrache l'image à son contexte sonore, la sépare de son ambiance habituelle et conformiste. Là encore il s'agit d'une esthétique du collage, du décalage et du contre-point. On a un exemple de ce type de travail dans une autre vidéo également analysée dans le livre : Barulho do silêncio (Le Bruit du silence). On y voit une série d'écrans qui constituent le fond d'une installation et sur lesquels s'anime l'agitation urbaine comme un mouvement brownien. À l'opposé de ce mur d'images, sur un autre plan, un écran présente un corps seul, presque immobile, qui contraste avec l'animation ambiante qui lui fait face. Cette image silencieuse symbolise l'impossibilité pour ce personnage solitaire de prendre part à l'effervescence de la vie urbaine. Sans commentaire, par le simple jeu des images en conflit, cette vidéo parle de l'inégalité face à la mobilité, économique, sociale, psychologique. Seul le dispositif est éloquent, constituant un appel à la sensibilité du spectateur.

Sonia Guggisberg aime à construire des situations de cette sorte, que le spectateur doit ensuite interpréter, qu'il doit relier à son propre vécu et aux circonstances dans lesquelles il s'est déjà trouvé. La poétique de l'image qui est mise en œuvre est une invitation à penser à travers la sensation. Ainsi, le travail de réflexion que mène l'auteure dans ce livre qui porte sur son propre travail ne cesse d'insister sur la contrepartie qu'offre à l'œuvre l'interprétation qui lui est apportée par le regardeur. Cela signifie que la proposition artistique est toujours ouverte dans la mesure où 
l'image est comme une introduction au champ des possibles et préserve la multiplicité des options cognitives. Même si l'artiste, dans sa tête, construit un projet de signification, la modalité de l'installation documentaire, à laquelle la vidéo donne forme et permanence, dépasse, par sa nature propre, le caractère déterminé de l'intention.

21 Sonia Guggisberg se débat de manière critique avec cette contradiction qu'on retrouve d'ailleurs dans le fait que la seule présence de la caméra dans l'espace de son objet cause une perturbation. Le documentaire n'est jamais le simple enregistrement de ce qui est : il intervient, perturbe, modifie le milieu où il s'introduit. Dans son rapport à son objet comme dans celui qui le lie à son spectateur, l'artiste doit construire sa place. Le rêve sucré de l'objectivité est aboli et la poétique mise en œuvre par l'artiste est la condition même du pouvoir performatif du documentaire. C'est du moins ce qu'on peut penser à la lecture de cet ouvrage qui représente un effort intellectuellement très fermement tenu. Ces essais, dans le dialogue qu'ils nouent avec les vidéos effectivement réalisées, constituent une solide base d'analyse des orientations esthétiques et politiques du documentaire vidéo d'aujourd'hui.

\section{NOTES}

1. Sonia Guggisberg, Redes de imagens, memórias e testemunhos : por uma documentação performativa de saberes, São Paulo, Intermeios - FAPESP, 2016, édition bilingue portugais et anglais. Titre en français de l'ouvrage: Réseaux d'images, mémoires et témoignages: pour une documentation performative des savoirs.

\section{AUTEUR}

JACQUES LEENHARDT

EHESS-CRAL, Paris 